\title{
High order transfer maps for perturbed Keplerian motion
}

\author{
Alexander Wittig ${ }^{1} \cdot$ Roberto Armellin ${ }^{2}$
}

Received: 14 October 2014 / Revised: 21 March 2015 / Accepted: 29 April 2015

(C) Springer Science+Business Media Dordrecht 2015

\begin{abstract}
The paper presents a new semi-analytical technique for the propagation of nearEarth satellite motion. The approach uses differential algebra techniques to compute the high order expansion of the solution of the system's ordinary differential equation for one orbital revolution, referred to as the transfer map. Once computed, a single high order transfer map (HOTM) can be reused to map an initial condition, or a set of initial conditions, forward in time for many revolutions. The only limiting factor is that the mapped objects must stay close to the reference orbit such that they remain within the region of validity of the HOTM. The performance of the method is assessed through a set of test cases in which both autonomous and non-autonomous perturbations are considered, including the case of continuously propelled trajectories.
\end{abstract}

Keywords Orbit propagation - Perturbed Keplerian motion - Differential algebra . High-order transfer map method

\section{Introduction}

Near-Earth satellite orbit propagation requires several sources of orbit perturbations (e.g. Earth non-sphericity, atmospheric drag, luni-solar perturbations, solar radiation pressure) to be included when modeling the dynamics. The techniques developed thus far for the solution of the perturbation problem fall into three broad categories: analytical, numerical, and semianalytical.

Roberto Armellin

roberto.armellin@soton.ac.uk

Alexander Wittig

alexander.wittig@polimi.it

1 Department of Aerospace Science and Technology, Politecnico di Milano, Via La Masa 34, 20156 Milan, Italy

2 Aeronautics, Astronautics and Computational Engineering Unit, University of Southampton, Highfield campus, Southampton SO17 1BJ, UK 
Analytical theories use perturbation theories based on various series expansions (Brouwer 1959; Kozai 1962; Lyddane 1963; Aksnes 1970; Kinoshita 1977; Hoots and Roehrich 1980; San-Juan 1994, 1998; Healy 2003) to transform the equations of motion such that closed form solutions are obtained. The solutions are explicit functions of time, initial conditions, and problem parameters; thus, the evaluation of an object's position and velocity state at a given time is reduced to the evaluation of explicitly known functions. Furthermore, analytical solutions are valid for all or at least large ranges of initial conditions. However, simplifications are necessary to obtain an analytical solution (e.g. simplified perturbation models and loworder approximations) which has a direct impact on the accuracy of the obtained solution.

Numerical techniques employ numerical integration of the equations of motion including all necessary perturbing accelerations. Numerical methods can give very high accurate results, as basically there are no restrictions on the details of the modeling of the perturbations. Thus, the results obtained with numerical propagation are often assumed as the truth (Long et al. 1989). The price to pay for this accuracy is that simulations can require high computational times due to the need of small integration step-sizes and must be repeated from the beginning for each different initial condition.

Semi-analytical methods have been developed to combine the efficiency of analytical methods and the accuracy of numerical integration. Within this approach, the highest frequencies of the motion, which normally have small amplitudes, are filtered analytically via averaging procedures. The averaged equations only depend on long period angles and, therefore, are integrated numerically with very large step sizes. The short-period effects, if required, can be recovered at any step of the integration by evaluation of the analytical expressions of the averaging. The averaging can be performed directly over the variation of parameters equations of motion using the generalized method of averaging (Bogoliuvov and Mitropolski 1961; McClain 1977), or in the Hamiltonian formulation of the dynamics using canonical perturbation theory (Hori 1966; Deprit 1969; Campbell and Jeffreys 1970; Métris and Exertier 1995; Lara et al. 2012). Independently of the method used, the derivation of the averaged equations is often a difficult and time consuming process: simplifications of the perturbations may be necessary and the approach may depend on the specific perturbation considered. Depending on the method, the type of perturbation that can be handled is limited, e.g. to Hamiltonian perturbations only (which shares some similarity with the multirevolution method by Graf and Bettis 1975)

We propose a new technique called high-order transfer map (HOTM) method. The technique, which appeared first in the field of particle accelerator physics (Berz 1987, 1999b), is here adapted to orbital dynamics exploiting the quasi-periodicity of the perturbed Keplerian motion. It is based on the automatic high order expansion of the solution of ordinary differential equations (ODEs) through differential algebra (DA). We consider this method semi-analytical in a broader sense as it, too, combines numerical and analytical techniques in the same method. However, unlike traditional semi-analytical methods, our proposed method reverses the order: first a HOTM is generated via a numerical integration of a single orbital revolution in DA arithmetic, yielding an analytical high order approximation of the true transfer map. It is then followed by its repeated analytical evaluation to advance the orbital propagation by several orbital periods. As the HOTM is obtained by the DA-based numerical integration of the equations of motion for a single revolution, the proposed method shares the mathematical simplicity of numerical techniques. On the other hand, as the bulk of the orbital propagation is achieved by multiple evaluations of the HOTM, which is represented as a high order Taylor polynomial, the method turns out to be numerically efficient like analytical methods. In addition, differently from other semi-analytical approaches, a HOTM is not based on averaging techniques and thus the osculating parameters are propagated. Moreover, 
the HOTM is valid in a neighborhood of the initial condition and therefore can be used to very efficiently propagate sets of initial conditions.

Of course the approach is not free of weaknesses. Typical perturbations cause a long-term drift in phase space. Due to this, the HOTM accuracy tends to degrade with the number of revolutions. After a certain number of revolutions, a new map has to be computed centered at the new state. Secondly, as a single orbital revolution is used to propagate the motion for several revolutions, the presence of highly non-autonomous perturbations can cause a rapid increase in approximation errors as the map converges only for a limited time range. Both limitations are discussed in detail later in this paper.

The remainder of the paper is organized as follows. First a description of our implementation of the perturbed dynamics is given. This is followed by a brief introduction on DA techniques and the explanation on how they enable the high-order expansion of the solution of ODEs with respect to both initial conditions and parameters. This is propaedeutical to the detailed description of the HOTM computation in Sect. 4. Test cases to assess the method's performance in the presence of autonomous (including low-thrust propulsion) and non-autonomous perturbations are presented in Sect. 5. Final remarks are made in Sect. 6.

\section{Dynamical model}

To avoid the singularities present in the equations of motion of the classical Keplerian orbital elements, we instead work with the set of modified equinoctial elements $\boldsymbol{x}=$ ( $p, f, g, h, k, L)$ (Walker et al. 1985; Walker 1986). The relationship between modified equinoctial elements and classical Keplerian elements is given by

$$
\begin{array}{lll}
p=a\left(1-e^{2}\right), & f=e \cos (\omega+\Omega), & g=e \sin (\omega+\Omega), \\
h=\tan (i / 2) \cos \Omega, & k=\tan (i / 2) \sin \Omega, & L=\Omega+\omega+\vartheta,
\end{array}
$$

where $a$ is the semi-major axis, $e$ the eccentricity, $i$ the inclination, $\Omega$ the right ascension of the ascending node, $\omega$ the argument of perigee, and $\vartheta$ the true anomaly. In the set of modified equinoctial elements $p$ is known as the semi-parameter and $L$ as true longitude.

The first-order system of differential equations describing the orbital motion in modified equinoctial elements is given by the following expressions

$$
\left\{\begin{array}{l}
\dot{p}=2 \sqrt{\frac{p}{\mu}} \frac{p}{q} \Delta_{t}=F_{p} \\
\dot{f}=\sqrt{\frac{p}{\mu}}\left(\sin L \Delta_{r}+\frac{1}{q}((1+q) \cos L+f) \Delta_{t}-\frac{g}{q}(h \sin L-k \cos L) \Delta_{n}\right)=F_{f} \\
\dot{g}=\sqrt{\frac{p}{\mu}}\left(-\cos L \Delta_{r}+\frac{1}{q}((1+q) \sin L+g) \Delta_{t}+\frac{f}{q}(h \sin L-k \cos L) \Delta_{n}\right)=F_{g} \\
\dot{h}=\sqrt{\frac{p}{\mu}} \frac{s^{2} \cos L}{2 q} \Delta_{n}=F_{h} \\
\dot{k}=\sqrt{\frac{p}{\mu}} \frac{s^{2} \sin L}{2 q} \Delta_{n}=F_{k} \\
\dot{L}=\sqrt{\frac{p}{\mu}} \frac{(h \sin L-k \cos L)}{q} \Delta_{n}+\sqrt{\mu p}\left(\frac{q}{p}\right)^{2}=F_{L},
\end{array}\right.
$$


in which $\dot{x}$ indicates the derivative of any modified equinoctial element $x$ with respect to time $t, \mu$ is the gravitational parameter of the Earth, $q=1+f \cos L+g \sin L, s^{2}=1+h^{2}+k^{2}$, and lastly $\boldsymbol{\Delta}=\left(\Delta_{r}, \Delta_{t}, \Delta_{n}\right)$ is the vector of perturbations expressed in the radial, transverse, and normal directions.

As the HOTM method is based on the numerical integration of a single orbital revolution it is preferable to work with $L$ as the independent variable. Since the equations of motion are periodic with respect to $L$, this allows the straightforward computation of a one revolution map (in the osculating elements) by integration from $L=0$ to $L=2 \pi$. The resulting map is also known as a Keplerian map.

Denoting by $x^{\prime}=\frac{\mathrm{d} x}{\mathrm{~d} L}$ the derivative of any modified equinoctial element $x$ with respect to $L$, we therefore apply the change of the independent variable according to $\dot{x}=\frac{\mathrm{d} x}{\mathrm{~d} L} \frac{\mathrm{d} L}{\mathrm{~d} t}=x^{\prime} F_{L}$ in the dynamics in Eq. (2). Furthermore, the last equation is replaced by the derivative of time $t$ with respect to $L$, resulting in the new set of equations

$$
\left\{\begin{array}{l}
p^{\prime}=F_{p} / F_{L} \\
f^{\prime}=F_{f} / F_{L} \\
g^{\prime}=F_{g} / F_{L} \\
h^{\prime}=F_{h} / F_{L} \\
k^{\prime}=F_{k} / F_{L} \\
t^{\prime}=1 / F_{L} .
\end{array}\right.
$$

For the remainder of this paper the state vector is given by $\boldsymbol{x}=(p, f, g, h, k, t)$ and $L$ is the independent variable.

The spacecraft position and velocity vectors $\boldsymbol{r}$ and $\boldsymbol{v}$ in the Earth centered inertial reference frame (ECI) are given by

$$
\boldsymbol{r}=\frac{r}{s^{2}}\left[\begin{array}{c}
\cos L+\alpha^{2} \cos L+2 h k \sin L \\
\sin L-\alpha^{2} \sin L+2 h k \cos L \\
2(h \sin L-k \cos L)
\end{array}\right]
$$

and

$$
\boldsymbol{v}=\frac{1}{s^{2}} \sqrt{\frac{\mu}{p}}\left[\begin{array}{c}
-\sin L-\alpha^{2} \sin L+2 h k \cos L-g+2 f h k-\alpha^{2} g \\
\cos L-\alpha^{2} \cos L-2 h k \cos L+f-2 g h k-\alpha^{2} f \\
2(h \cos L+k \sin L+f h+g k)
\end{array}\right],
$$

in which $q$ and $s$ are as before and $r=p / q$ and $\alpha^{2}=h^{2}-k^{2}$.

The unit vectors for the radial, transverse, and normal directions are then given by

$$
\hat{\boldsymbol{r}}=\frac{r}{r}, \quad \hat{\boldsymbol{n}}=\frac{\boldsymbol{r} \times \boldsymbol{v}}{\|\boldsymbol{r} \times \boldsymbol{v}\|}, \quad \hat{\boldsymbol{t}}=\hat{\boldsymbol{n}} \times \hat{\boldsymbol{r}},
$$

respectively. Together they define the radial, transverse, and normal reference frame (RTN). The transformation from RTN to ECI is given by the rotation matrix $\mathbf{T}=[\hat{\boldsymbol{r}}, \hat{\boldsymbol{t}}, \hat{\boldsymbol{n}}]$.

In the examples presented in this work we will consider the contribution of different sources of perturbation to demonstrate the capability of the HOTM method to efficiently handle both non-autonomous and autonomous dynamics, as well as continuously propelled arcs. A short description of the implementation of these perturbations is given in the following 
subsections. Note that simple perturbation models are adopted as the main goal of the work is to demonstrate the HOTM concept rather than developing a complete orbital propagator.

\subsection{Non-spherical Earth gravity}

In this work only the contribution of the zonal harmonic $J_{2}$ is taken into account. The acceleration due to $J_{2}$ is expressed directly in modified equinoctial elements in the RTN reference frame as

$$
\left\{\begin{array}{l}
\Delta_{J_{2 r}}=-\frac{3 \mu J_{2} R_{E}^{2}}{2 r^{4}}\left(1-\frac{12(h \sin L-k \cos L)^{2}}{\left(1+h^{2}+k^{2}\right)^{2}}\right) \\
\Delta_{J_{2 t}}=-\frac{12 \mu J_{2} R_{E}^{2}}{r^{4}}\left(\frac{(h \sin L-k \cos L)(h \cos L+k \sin L)}{\left(1+h^{2}+k^{2}\right)^{2}}\right) \\
\Delta_{J_{2 n}}=-\frac{6 \mu J_{2} R_{E}^{2}}{r^{4}}\left(\frac{\left(1-h^{2}-k^{2}\right)(h \sin L-k \cos L)}{\left(1+h^{2}+k^{2}\right)^{2}}\right),
\end{array}\right.
$$

in which $R_{E}$ is the equatorial radius of the Earth.

\subsection{Third body perturbation}

The contribution of accelerations due to third bodies in the ECI reference frame is given by

$$
\boldsymbol{\Delta}_{3 b_{E C I}}=-\sum_{j=1}^{n} \mu_{j}\left(\frac{\boldsymbol{d}_{j}}{d_{j}^{3}}+\frac{\boldsymbol{s}_{j}}{s_{j}^{3}}\right),
$$

where $\mu_{j}$ is the gravitational constant of each perturbing body, $\boldsymbol{s}_{j}$ is the vector from the primary body to the secondary body, and $\boldsymbol{d}_{j}=\boldsymbol{r}-\boldsymbol{s}_{j}$ is the position vector of the spacecraft relative to the secondary body while $s_{j}$ and $d_{j}$ refer to their Euclidean norms. The expression of the third body perturbation is inserted into Eq. (2) after rotation into the RTN reference frame by the transformation

$$
\boldsymbol{\Delta}_{3 b_{R T N}}=\mathbf{T}^{T} \boldsymbol{\Delta}_{3 b_{E C I}} .
$$

Simplified ephemerides, in which the orbital parameters of the gravitational bodies are expressed as low order polynomials in time (maximum order 4) are used in this work. In the test cases presented in this paper we also account for luni-solar perturbations.

\subsection{Atmospheric drag}

The radial, tangential, and normal accelerations due to aerodynamics drag are given by

$$
\left\{\begin{array}{l}
\Delta_{D_{r}}=-\frac{1}{2} C_{D} \frac{A}{m} \rho v v_{r} \\
\Delta_{D_{t}}=-\frac{1}{2} C_{D} \frac{A}{m} \rho v v_{t}, \\
\Delta_{D_{n}}=0,
\end{array}\right.
$$

where $A$ and $m$ are the satellite's cross-sectional area and mass respectively, $C_{D}$ is the drag coefficient, and $v$ is the magnitude of the velocity. The radial and transversal components of 
the velocity, $v_{r}$ and $v_{t}$, are given by

$$
v_{r}=\sqrt{\frac{\mu}{p}}(f \sin L-g \cos L), \quad v_{t}=\sqrt{\frac{\mu}{p}}(1+f \cos L+g \sin L) .
$$

The atmospheric density $\rho$ is modeled as a function of the altitude $h$ as

$$
\rho(h)=\exp \left(c_{0}+c_{1} h+c_{2} h^{2}+c_{3} h^{3}+c_{4} h^{4}\right),
$$

in which the coefficients $c_{i}$ are obtained by means of fitting the density obtained from the Naval Research Laboratory's Mass Spectrometer and Incoherent Scatter Radar of the year 2000 (NRLMSISE-00) model (Picone et al. 2002). The fit was performed at a fixed epoch, for $h \in[200,1000] \mathrm{km}$, and averaging the results at different longitudes and latitudes. The values thusly obtained and used for the test cases presented in this paper are $c_{0}=-15.91, c_{1}=$ $-0.03884, c_{2}=4.33 \times 10^{-5}, c_{3}=-4.097 \times 10^{-8}$, and $c_{4}=1.858 \times 10^{-11}$. The units of these coefficients are such that the evaluation of Eq. 12 with $h$ in $\mathrm{km}$ returns the atmospheric density in $\mathrm{kg} / \mathrm{m}^{3}$.

\subsection{Solar radiation pressure}

The contribution due to solar radiation pressure is approximated in the ECI reference frame as

$$
\boldsymbol{\Delta}_{S R P_{E C I}}=P_{S} C_{R} \frac{A}{m} \frac{\boldsymbol{d}_{S}}{d_{S}{ }^{3}},
$$

where, as for the third body perturbation, $\boldsymbol{d}_{S}$ is the position vector of the satellite relative to the Sun and $d_{S}$ its Euclidean norm. The solar pressure $P_{S}$ is obtained by dividing the flux $\Phi \approx 1367 \mathrm{~W} / \mathrm{m}^{2}$ at $1 \mathrm{AU}$, by the speed of light $c=299792458 \mathrm{~m} / \mathrm{s}$. Finally, the spacecraft is modeled as a sphere and $C_{R}$ is the radiation pressure coefficient which accounts for the mean reflectivity of its surface (Montenbruck and Gill 2000).

As for the third-body perturbation, the contribution of the solar radiation pressure perturbation is inserted into Eq. (2) after rotation into the RTN reference frame by the transformation

$$
\boldsymbol{\Delta}_{S R P_{R T N}}=\mathbf{T}^{T} \boldsymbol{\Delta}_{S R P_{E C I}}
$$

In this first work on the topic, solar eclipses are not yet modeled.

\subsection{Thrust}

The action of a low-thrust propulsive system is modeled as

$$
\boldsymbol{\Delta}_{P_{R T N}}=\Delta_{P_{R T N}} \hat{\boldsymbol{p}}
$$

where $\Delta_{P_{R T N}}$ is the thrust acceleration magnitude and $\hat{\boldsymbol{p}}$ its direction in the RTN reference frame. Both thrust direction and magnitude can be arbitrary functions of the state vector. In this paper, we model the thrust direction as either fixed in the RTN reference frame (i.e., $\hat{\boldsymbol{p}}$ is constant), or aligned with the velocity vector whose RTN components are given by Eq. (11). The thrust magnitude $\Delta_{P_{R T N}}$ is considered constant during each orbital revolution. 
Fig. 1 Analogy between the floating point representation of real numbers in a computer environment (left) and the introduction of the algebra of Taylor polynomials in the differential algebraic framework (right)
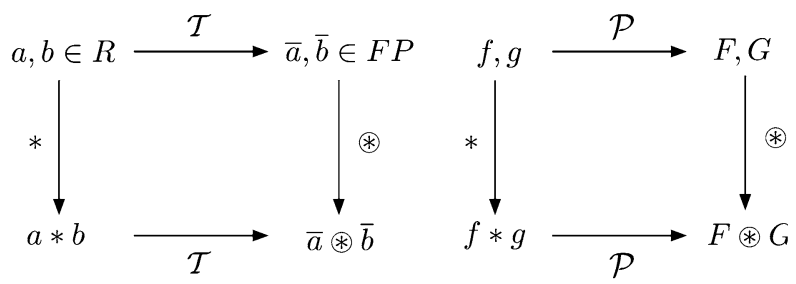

\section{Notes on differential algebra}

Differential Algebra techniques were devised to attempt solving analytical problems through an algebraic approach (Berz 1999b). Historically, the treatment of functions in numerics has been based on the treatment of numbers, and the classical numerical algorithms are based on the mere evaluation of functions at specific points. DA techniques rely on the observation that it is possible to extract more information on a function rather than its mere values. The basic idea is to bring the treatment of functions and the operations on them to computer environment in a similar manner as the treatment of real numbers. Referring to Fig. 1, consider two real numbers $a$ and $b$. Their transformation into the floating point representation, $\bar{a}$ and $\bar{b}$ respectively, is performed to operate on them in a computer environment. Then, given any operation $*$ in the set of real numbers, an adjoint operation $\circledast$ is defined in the set of floating point (FP) numbers so that the diagram in Fig. 1 commutes. (The diagram commutes approximately in practice due to truncation errors). Consequently, transforming the real numbers $a$ and $b$ into their FP representation and operating on them in the set of FP numbers returns the same result as carrying out the operation in the set of real numbers and then transforming the achieved result in its FP representation. In a similar way, let us suppose two $k$ differentiable functions $f$ and $g$ in $n$ variables are given. In the framework of differential algebra, the computer operates on them using their $k$-th order Taylor expansions, $F$ and $G$ respectively. Therefore, the transformation of real numbers in their FP representation is now substituted by the extraction of the $k$-th order Taylor expansions of $f$ and $g$. For each operation in the space of $k$ differentiable functions, an adjoint operation in the space of Taylor polynomials is defined so that the corresponding diagram commutes; i.e., extracting the Taylor expansions of $f$ and $g$ and operating on them in the space of Taylor polynomials (labeled as ${ }_{k} D_{n}$ ) returns the same result as operating on $f$ and $g$ in the original space and then extracting the Taylor expansion of the resulting function.

The straightforward implementation of differential algebra in a computer allows computation of the Taylor coefficients of a function up to a specified order $k$, along with the function evaluation, with a fixed amount of effort. The Taylor coefficients of order $n$ for sums and products of functions, as well as scalar products with reals, can be computed from those of summands and factors; therefore, the set of equivalence classes of functions can be endowed with well-defined operations, leading to the so-called truncated power series algebra (Berz 1986, 1987). Similarly to the algorithms for floating point arithmetic, the algorithms for functions followed, including methods to perform composition of functions, to invert them, to solve nonlinear systems explicitly, and to treat common elementary functions (Berz 1999a, b). In addition to these algebraic operations, the DA framework is endowed with differentiation and integration operators, therefore finalizing the definition of the DA structure. The DA sketched in this section was implemented by Berz and Makino in the software COSY INFINITY (Berz and Makino 2006). 


\subsection{High-order expansion of the solution of an ODE}

Differential algebra allows the derivatives of any function $f$ of $n$ variables to be computed up to an arbitrary order $k$, along with the function evaluation. This has an important consequence when the numerical integration of an ODE is performed by means of an arbitrary integration scheme. Any integration scheme is based on algebraic operations, involving the evaluation of the ODE right hand side at several integration points. Therefore, carrying out all the evaluations in the DA framework allows differential algebra to compute the arbitrary order expansion of the flow of a general ODE with respect to the initial condition.

Without loss of generality, consider the scalar initial value problem (IVP)

$$
\left\{\begin{array}{l}
\dot{x}=f(x, t) \\
x\left(t_{0}\right)=x_{0}
\end{array} .\right.
$$

We now want to show that, starting from the DA representation of the initial condition $x_{0}$, differential algebra allows us to compute the Taylor expansion of the IVP with respect to the initial condition at the final time $t_{f}$.

Replace the point initial condition $x_{0}$ by the DA representative of its identity function up to order $k$, which is a $(k+1)$-tuple of Taylor coefficients. As only the first two coefficients, corresponding to the constant part and the first derivative respectively, are non zeros, we can write the DA variable $\left[x_{0}\right]$ as $x_{0}+\delta x_{0}$, in which $x_{0}$ is the reference point for the expansion. If all the operations of the numerical integration scheme are carried out in the framework of differential algebra, the solution $x_{i}$ is approximated, at each fixed time step $t_{i}$, as a Taylor expansion in $x_{0}$.

For the sake of clarity, consider the forward Euler's scheme

$$
x_{i}=x_{i-1}+f\left(x_{i-1}\right) \Delta t
$$

and substitute the initial value with the DA identity $\left[x_{0}\right]=x_{0}+\delta x_{0}$. At the first time step we have

$$
\left[x_{1}\right]=\left[x_{0}\right]+f\left(\left[x_{0}\right]\right) \cdot \Delta t .
$$

If the function $f$ is evaluated in the DA framework, the output of the first step, $\left[x_{1}\right]$, is the $k$-th order Taylor expansion of the solution of the IVP in $x_{0}$ at $t=t_{1}$. Note that, as a result of the DA evaluation of $f\left(\left[x_{0}\right]\right)$, the $(k+1)$-tuple $\left[x_{1}\right]$ may include several non zeros coefficients corresponding to high-order terms in $\delta x_{0}$. The previous procedure can be inferred through the subsequent steps. The result of the final step is the $k$-th order Taylor expansion of the solution in $x_{0}$ at the final time $t_{f}$. Thus, the solution of the IVP can be approximated, at each time step $t_{i}$, as a $k$-th order Taylor expansion in $x_{0}$ in a fixed amount of effort. In the reminder of the paper we will express this result as $\left[x_{i}\right]=\mathcal{M}_{x_{0}}\left(\delta x_{0}\right)$, in which the square brackets reminds that the output is a DA variable, $\mathcal{M}$ indicates a Taylor map or polynomial, the subscript that the variables of the Taylor expansion, and the $\delta$ remind that the Taylor expansion is function of the variation with respect to the reference values.

Note that the expansion of the solution of the IVP can be easily obtained also with respect to any parameter $q$ that appears in the dynamics model. In this case also the parameter $p$ has to be initialized as a DA variable, i.e. $[q]=q+\delta q$, and the solution at time $t_{i}$ is $\left[x_{i}\right]=\mathcal{M}_{x_{0}, q}\left(\delta x_{0}, \delta q\right)$.

The conversion of standard integration schemes to their DA counterparts is straightforward for explicit solvers, substituting operations between real numbers with those on DA objects. In addition, whenever the integration scheme involves step size control, an appropriate measure of the accuracy of the Taylor expansion of the flow needs to be included. 
The main advantage of the DA-based approach is that there is no need to write and integrate variational equations in order to obtain high order expansions of the flow. This result is basically obtained by the substitution of operations between real numbers with those on DA numbers, and therefore the method is ODE independent. Furthermore, the efficient implementation of the differential algebra in COSY-Infinity allows us to obtain high order expansions with limited computational time.

In previous applications by the authors (Valli et al. 2013; Morselli et al. 2014) the map $\left[x_{i}\right]=\mathcal{M}_{x_{0}, q}\left(\delta x_{0}, \delta q\right)$ has been used to efficiently propagate set of initial conditions by replacing intensive Monte Carlo simulations (based on multiple integration of ODE) with multiple evaluations of the Taylor map. In this work the availability of the Taylor map, combined with the quasi-periodicity of perturbed Keplerian motion, is exploited to implement an efficient method for orbital propagation.

\section{HOTM method}

The HOTM method is applicable to a wide variety of semi-periodic systems. In particular, it can be applied to the dynamics introduced in Sect. 2 with both autonomous and non-autonomous perturbations. For the sake of clarity, we will fist briefly introduce the mathematical foundation of the HOTM method in general, and then discuss the particular application of the method to orbit propagation with both autonomous and non-autonomous perturbations.

\subsection{Mathematical foundation of the HOTM}

The basic idea of the method is to replace numerical integration by the repeated evaluation of a discrete map representing the dynamics of the system, exploiting the periodicity of the dynamics.

Consider a dynamical system defined by the ODE

$$
\frac{d \boldsymbol{x}}{d y}=f(\boldsymbol{x}, y)
$$

where the right hand side $f(\boldsymbol{x}, y)$ is periodic in $y$ with period $P$, i.e.

$$
f(\boldsymbol{x}, y)=f(\boldsymbol{x}, y+P)
$$

for all $\boldsymbol{x}$ and $y$ and $f$ is at least Lipschitz continuous in $\boldsymbol{x}$ and continuous in $y$ over the domain of interest. A typical case for such a dynamical system is a system parametrized by an angle such as, for example, the system in (3).

Let $\varphi_{x}(y)$ be the solution of (19) such that $\varphi_{\boldsymbol{x}}(0)=\boldsymbol{x}$. From the periodicity condition (20) it follows that $g(y)=\varphi_{x}(y+P)$ is a solution of (19) satisfying $g(0)=\varphi_{x}(P)$. By the Picard-Lindelöf theorem we have uniqueness of the solution and hence

$$
\varphi_{x}(y+P)=g(y)=\varphi_{\varphi_{x}(P)}(y) .
$$

Given an initial state $\boldsymbol{x}_{0}$, consider now the sequence $\boldsymbol{x}_{i}$ defined by

$$
\boldsymbol{x}_{i}=\varphi_{x_{0}}(i \cdot P),
$$

which represents the final state of $\boldsymbol{x}_{0}$ after $i$ periods of $y$. By (21) we can rewrite (22) in recursive form as

$$
\boldsymbol{x}_{i+1}=\varphi_{\boldsymbol{x}_{i}}(P)
$$


Fig. 2 Illustration of the map $\Phi$ for the case of $x \in \mathbb{R}^{2}$. Snapshots of the phase space are shown at $y=0, y=P$, and $y=2 P$, along with the continuous trajectory $\boldsymbol{x}(y)$. Neighboring snapshots are related by the map $\Phi: \mathbb{R}^{2} \rightarrow \mathbb{R}^{2}$

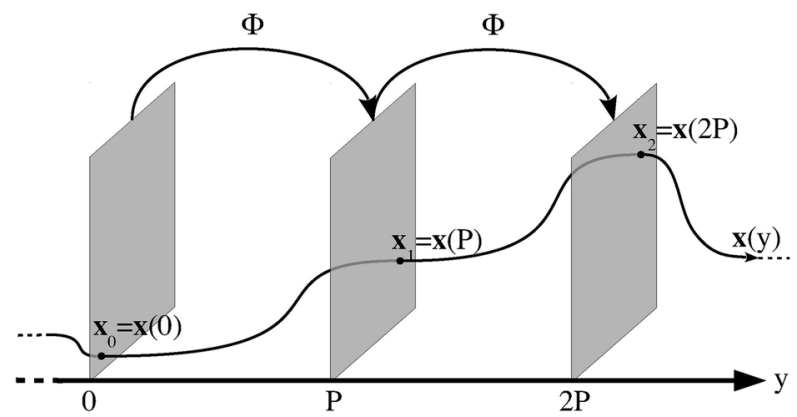

It is this sequence of states $\boldsymbol{x}_{i}$ we want to compute when we perform orbit propagation.

Thus, introducing the function

$$
\Phi(\boldsymbol{x})=\varphi_{\boldsymbol{x}}(P)
$$

the problem is now to find an efficient method to calculate $\Phi$. Once we have this, we can use it to compute all iterates $\boldsymbol{x}_{i}$ directly. The situation is illustrated in Fig. 2. Note that $\Phi(\boldsymbol{x})$ is simply the solution of the ODE at $y=P$ for the initial condition $x$ at $y=0$.

In practice, the difficulty lies in the actual computation of the map $\Phi$. Only in specific simple cases is it possible to calculate an analytic expression for $\Phi$. The most common approach to computing it in the general case is numerical integration. Unfortunately, while this allows the pointwise evaluation of $\Phi$, it requires a computationally intensive numerical integration to be carried out for every single evaluation.

Instead, in our method we use DA to compute a computer representation of the function $\Phi$ as a high-order polynomial expansion $\mathcal{M}_{\boldsymbol{x}_{0}}$ around $\boldsymbol{x}_{0}$ such that

$$
\mathcal{M}_{x_{0}}\left(\delta x_{0}\right)=\Phi\left(x_{0}+\delta x_{0}\right) .
$$

This is done by initializing the initial condition as a DA variable $\left[\boldsymbol{x}_{\mathbf{0}}\right]=\boldsymbol{x}_{\mathbf{0}}+\boldsymbol{\delta} \boldsymbol{x}_{0}$ and performing the integration from $y=0$ to $y=P$ using a DA based integration scheme (see Sect. 3.1). This process yields a DA expansion $\left[\boldsymbol{x}_{1}\right]=\mathcal{M}_{\boldsymbol{x}_{0}}\left(\boldsymbol{\delta} \boldsymbol{x}_{0}\right)$ in terms of $\boldsymbol{\delta} \boldsymbol{x}_{0}$ around the reference orbit $\boldsymbol{x}_{0}$.

The specific integration scheme selected to perform the numerical integrations in this work is a DA implementation of a standard Dormand-Prince 7/8 integrator of the Runge-Kutta family with an 8-th order solution for propagation and 7-th order solution used for step size control.

We call (23) a high order transfer map (HOTM). The HOTM $\mathcal{M}_{\boldsymbol{x}_{0}}$ is a computer representation of the function $\Phi(\boldsymbol{x})$. The initial computation of $\mathcal{M}$ using DA is typically much more computationally expensive than the numerical integration of a single initial condition for one orbital revolution. However, once computed it is represented as a polynomial, and the repeated evaluation of the map becomes trivial. In particular, there is no need for further integrations to evaluate $\mathcal{M}_{x_{0}}$. We also note that this greatly reduces the requirements on the computational environment, as polynomial evaluation requires only multiplication and addition. It can therefore be easily implemented even on very restricted hardware.

We can now use this fact to efficiently compute approximations $\widetilde{\boldsymbol{x}}_{1}, \widetilde{\boldsymbol{x}}_{2}, \ldots$ to the sequence $\boldsymbol{x}_{1}, \boldsymbol{x}_{2}, \ldots$ given by

$$
\widetilde{\boldsymbol{x}}_{i}=\mathcal{M}_{\boldsymbol{x}_{\mathbf{0}}}\left(\widetilde{\boldsymbol{x}}_{i-1}-\boldsymbol{x}_{0}\right) \text {. }
$$


Fig. 3 Illustration of the action of the map $\Phi$ in the case of unperturbed Keplerian motion (a) and with small perturbations (b), with the projection of the first two iterates $\boldsymbol{x}_{1}, \boldsymbol{x}_{2}$ into the $p-f$ plane. In $\mathbf{b}$ also a radius of convergence is shown

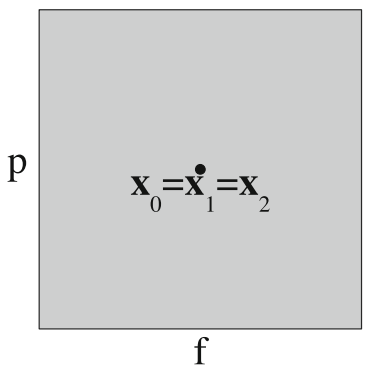

(a)

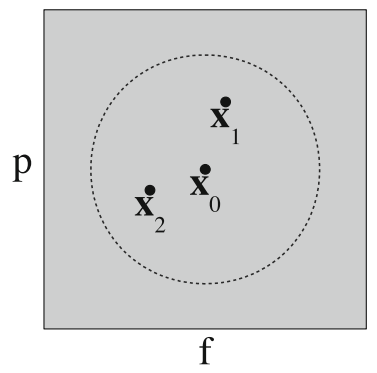

(b)

Being a polynomial expansion of the true map expanded around a given initial state $\boldsymbol{x}_{0}$, the HOTM $\mathcal{M}$ has some radius of convergence around the expansion point $\boldsymbol{x}_{0}$ within which it will accurately represent the original map. The size of this radius depends strongly on the dynamics and the period $P$, the expansion point, and the expansion order of the polynomial. The problem of obtaining an estimate for radius of convergence is addressed in Sect. 4.5.

\subsection{Autonomous perturbations}

We now apply the HOTM method specifically to the dynamics defined in Eq. (3) with only autonomous perturbations. As the equations of motion for $p, f, g, h, k$ in Eq. (3) decouple from the motion of $t$, for a first discussion we can consider only the reduced state vector $\boldsymbol{x}=(p, f, g, h, k)$, while ignoring time evolution.

Since the variable $L$ represents an angle in modified equinoctial elements, the dynamics are periodic in $L$ with period $P=2 \pi$ as required by Eq. (20). The sequence $\boldsymbol{x}_{i}$ defined in Eq. (22) represents the state of the spacecraft after $i$ revolutions in the osculating modified equinoctial elements. That is

$$
\boldsymbol{x}_{i}=\boldsymbol{x}(L=2 \pi i)
$$

In pure Keplerian motion the modified equinoctial elements $p, f, g, h, k$ are constants of motion. Thus for $\boldsymbol{\Delta}=\mathbf{0}$ in Eq. (3), the map $\Phi$ is represented by the identity

$$
\Phi(x)=x
$$

and hence the HOTM $\mathcal{M}$ is given by

$$
\mathcal{M}_{x_{0}}\left(\delta x_{0}\right)=x_{0}+\delta x_{0}
$$

This situation is illustrated in Fig. 3a.

We make the following observations:

1. Because $\Phi$ is a linear function (the identity), the polynomial expansion $\mathcal{M}$ is exact and converges everywhere;

2. The iterates of $\boldsymbol{x}_{i}$ stay close to $\boldsymbol{x}_{0}$, in fact $\boldsymbol{x}_{i}=\boldsymbol{x}_{0}$;

3. Since $\mathcal{M}$ is exact and has no truncation error, we have that $\widetilde{\boldsymbol{x}}_{i}=\boldsymbol{x}_{i}=\boldsymbol{x}_{0}$.

As we move to perturbed Keplerian motion, all of these properties disappear. $\Phi$ becomes non-linear, and the HOTM $\mathcal{M}_{\boldsymbol{x}_{0}}$ only converges in some neighborhood of $\boldsymbol{x}_{0}$. The $\boldsymbol{x}_{i}$ are not constant any more, and due to the truncation error in $\mathcal{M}$ in general $\widetilde{\boldsymbol{x}}_{i} \neq \boldsymbol{x}_{i}$. However, since the perturbations $\boldsymbol{\Delta}$ are small, it is reasonable to assume that the iterates $\boldsymbol{x}_{i}$ remain close to $\boldsymbol{x}_{0}$ for some number of revolutions, as illustrated in Fig. $3 \mathrm{~b}$. This ensures that the map iterates 
$\widetilde{\boldsymbol{x}}_{i}$ remain within the map's radius of convergence, which in turn ensures that the sequence $\widetilde{\boldsymbol{x}}_{i}$, if not exact, is at least an accurate approximation of $\boldsymbol{x}_{i}$.

We now return to the question of time evolution and the full state vector $\boldsymbol{x}=$ ( $p, f, g, h, k, t)$. Unfortunately, time of course is monotonously increasing with $L$, and thus the time component $t_{i}$ of the iterates $\boldsymbol{x}_{i}$ never stays close to $t_{0}$. Fortunately, in the case of autonomous perturbations this has absolutely no effect on the accuracy of the method as the HOTM $\mathcal{M}$ converges for all $t$, as explained in the following.

First, consider again the case of purely Keplerian motion. Here the time $T$ for one full revolution in $\vartheta$ (and since $\Omega$ and $\omega$ are constant equivalently in $L=\Omega+\omega+\vartheta$ ) is given by

$$
2 \pi \sqrt{a^{3} / \mu}=2 \pi \sqrt{\left(\frac{p}{1-f^{2}-g^{2}}\right)^{3} / \mu}=T(p, f, g)
$$

and depends non-trivially on $p, f, g$. Thus the complete function $\Phi(\boldsymbol{x})$ is given by

$$
\Phi(\boldsymbol{x})=\Phi\left(\left(\begin{array}{l}
p \\
f \\
g \\
h \\
k \\
t
\end{array}\right)\right)=\left(\begin{array}{c}
p \\
f \\
g \\
h \\
k \\
t+T(p, f, g)
\end{array}\right) .
$$

When computing the HOTM $\mathcal{M}_{\boldsymbol{x}_{0}}$, which is the Taylor expansion of $\Phi$ around $\boldsymbol{x}_{0}$, the non-trivial dependence $T(p, f, g)$ is automatically expanded as a Taylor series around the reference values $p_{0}, f_{0}, g_{0}$. As the dependence is non-polynomial, this expansion has a limited radius of convergence around $p_{0}, f_{0}, g_{0}$. As a result, while the non-time elements of $\widetilde{\boldsymbol{x}}_{i}$ in the case of unperturbed motion are always propagated exactly, the time component $\widetilde{t}_{i}$ even in the unperturbed case, is accurate only if $p, f, g$ are close to $p_{0}, f_{0}, g_{0}$ respectively.

An important observation, however, is that $\Phi$ is linear in the remaining elements, $h, k$, and $t$, and thus the Taylor expansion $\mathcal{M}$ is exact for any value of $h, k, t$. In particular, this means that the radius of convergence of $\mathcal{M}$ in $t$ is infinite, i.e. the map converges for all $t$. As long as $p_{i}, f_{i}, g_{i}$ are near $p_{0}, f_{0}, g_{0}$ respectively, even if $t_{i}$ is already far from $t_{0}$ the map iterate $\tilde{t}_{i+1}$ will still be an accurate approximation of $t_{i+1}$. This situation is illustrated in Fig. $4 \mathrm{a}$.

Moving from the unperturbed to autonomously perturbed dynamics does not change the situation of time propagation much. Now that perturbations are included, the period $T$ for one revolution in $L$ will not be just a function of $p, f, g$ any more, but depend on all the non-time elements $p, f, g, h, k$. However, as the dynamics are autonomous, the time $t$ itself does not influence the period $T$. This important observation means that only the time component of $\Phi$ retains a (linear) dependence on $t$ and hence $\mathcal{M}$ still converges for all $t$. This situation is illustrated in Fig. 4b.

\subsection{Non-autonomous perturbations}

As has been demonstrated in the previous section, in the case of autonomous dynamics the accuracy of the HOTM method is not affected by the times $t_{i}$, or their distance from $t_{0}$. Unfortunately, once non-autonomous perturbations are considered this is not the case any more. Once the perturbations $\boldsymbol{\Delta}$ depend explicitly on the time $t$, all components of $\Phi$ have an explicit, non-linear time dependence and thus also the HOTM $\mathcal{M}_{\boldsymbol{x}_{0}}$ is nonlinear in $t$. Taken by itself this is no problem, the HOTM $\mathcal{M}_{x_{0}}$ still represents a good approximation of $\Phi$ in some neighborhood around $\boldsymbol{x}_{0}$. In particular, the map is accurate in some time interval $t_{0} \pm r_{t}$. 
Fig. 4 Illustration of the action of the map $\Phi$ in the case of unperturbed Keplerian motion (a) and with small perturbations (b), with the projection of the first two iterates $\boldsymbol{x}_{1}, \boldsymbol{x}_{2}$ into the $p-t$ plane. In both cases also the radius of convergence is shown, which becomes a strip as the radius of convergence in $t$ is infinite

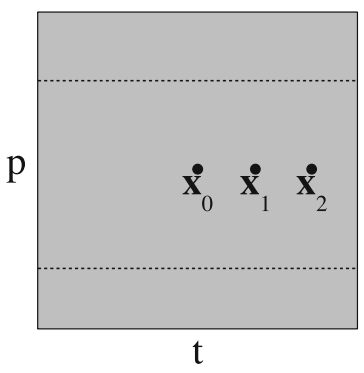

(a)

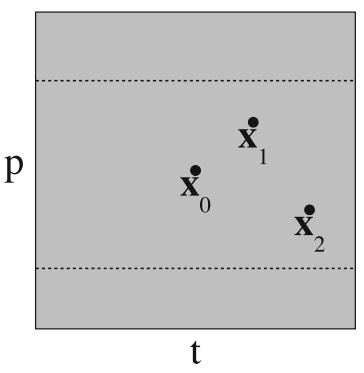

(b)

However, unlike the non-time elements, time is monotonously increasing and unbounded. Thus for any dynamics there will be a number of revolutions $N_{\max }$ such that $t_{i}-t_{0}>r_{t}$ for all $i>N_{\max }$, and consequently independently of the other elements the iterates $\tilde{\boldsymbol{x}}_{i}$ can only be accurate up to $i=N_{\max }$. Depending on the size of the radius of convergence $r_{t}$ as well as the period $T$, this may significantly limit the number of revolutions the map can be used for.

The typical period $T$ of an orbit around Earth is on the order of hours or at most days. The size of the radius of convergence $r_{t}$, on the other hand, is determined by the specific time-dependence of the perturbation. If the time dependence is weak, that is the perturbation only changes very little over one revolution, then the Taylor expansion of the perturbation will converge well over large time spans.

This is the case, for example, with perturbations related to the Sun such as solar radiation pressure or solar gravity. The period of the motion of the Earth around the Sun is one year, the Taylor expansion of the Earth ephemeris therefore will converge well over many revolutions. However, the situation is already different for the perturbation due to the Moon. The Moon's period is only about 28 days, and the perturbation due to the Moon position changes much more quickly than that due to the Sun. The Taylor expansion of the lunar perturbation therefore only converges over a smaller time range $r_{t}$ and may be the limiting factor in determining the number of revolutions a map can be used for.

The main way to increase the time validity of the map is to increase the expansion order of the HOTM. However, in order to save computational time, instead of computing the entire map up to higher order in all variables, only the order of the time variable $t$ is increased. The effect is that the radius of convergence $r_{t}$ for time $t$ increases, while the (already sufficient) radius of convergence for the other variables remains unchanged. In the test case presented in this work we select order 4 or 5 for spacial variables and order 12 for time. This choice is motivated by the observations from the autonomous case and the technical restriction of the COSY INFINITY DA implementation that requires weighted orders to be odd multiples of each other.

As a last remark, it has to be stressed that the choice of the expansion point plays a key role in maximizing the utility of a HOTM in the presence of non-autonomous perturbations. Instead of expanding around the initial time $t_{0}$, it is beneficial to expand around time $t_{0}+r_{t}$. The reason is that the convergence radius of a polynomial expansion is typically symmetric. That is, the polynomial expansion around $t^{*}$ will converge in an interval of the form $\left[t^{*}-\right.$ $\left.r_{t}, t^{*}+r_{t}\right]$. Choosing $t^{*}=t_{0}$, we therefore obtain a Taylor expansion converging on $\left[t_{0}-\right.$ $\left.r_{t}, t_{0}+r_{t}\right]$. However since time is monotonously increasing, we will never evaluate the left half of the interval $\left[t_{0}-r_{t}, t_{0}\right]$ and the usable interval is limited to $\left[t_{0}, t_{0}+r_{t}\right]$. If, on the other hand, we choose $t^{*}=t_{0}+r_{t}$ the interval in which the expansion converges becomes 
$\left[t_{0}+r_{t}-r_{t}, t_{0}+r_{t}+r_{t}\right]=\left[t_{0}, t_{0}+2 r_{t}\right]$, thus doubling the time for which we can use the map. Of course $r_{t}$ typically is not known exactly ahead of time, but a rough estimate can be obtained as outlined in Sect. 4.5.

\subsection{Advanced HOTM techniques}

In this section we briefly introduce two advanced features, namely the capability of mapping many initial conditions and of enabling sensitivity analyses with respect to system parameters, that can contribute to widen the set of applications of the HOTM method.

\subsubsection{Propagation of set of initial conditions}

An important feature of the HOTM method, both for autonomous and non-autonomous dynamics, is that there is no need to recompute the HOTMs if new initial conditions $\boldsymbol{x}_{0}^{j}$ (with $j=1, \ldots, M)$ need to be propagated. For each initial condition $\widetilde{\boldsymbol{x}}_{0}^{j}=\boldsymbol{x}_{0}^{j}$, the associated state after $i$ orbital revolutions is obtained by the recursive definition

$$
\tilde{\boldsymbol{x}}_{i}^{j}=\mathcal{M}_{\boldsymbol{x}_{\mathbf{0}}}\left(\widetilde{\boldsymbol{x}}_{i-1}^{j}-\boldsymbol{x}_{0}\right) .
$$

Thus, for any additional initial conditions the orbit propagation is reduced to the fast evaluation of (25). As before, in order for the iterates $\widetilde{\boldsymbol{x}}_{i}^{j}$ to be accurate it is necessary that all iterates, and in particular the initial condition $\widetilde{\boldsymbol{x}}_{0}^{j}$, lie within the radius of convergence of $\mathcal{M}_{\boldsymbol{x}_{0}}$. That is to say, the initial conditions $\boldsymbol{x}_{0}^{j}$ must be close to $\boldsymbol{x}_{0}$.

Consequently, this feature is particularly relevant when entire sets of similar initial conditions need to be propagated, as is the case for sensitivity analysis, state estimation, or debris-cloud evolution. As the examples in Sect. 5 show, the computational time to evaluate the map $\mathcal{M}_{\boldsymbol{x}_{0}}$ is negligible compared to numerical integration, allowing the propagation of clouds of hundreds of thousands of points at virtually no computational cost.

\subsubsection{Expansion with respect to parameters}

When running sensitivity analysis it is often required to assess the sensitivity of a system not only to initial condition, but also to model parameters, as for example atmospheric density. Furthermore, in the study of debris-cloud evolution, each initial condition corresponds to a different physical object, characterized for example by a different value of $A / m$. The HOTM method can be easily adapted to efficiently deal with such situations.

As explained in Sect. 4.1, the HOTM $\mathcal{M}_{\boldsymbol{x}_{0}}$ is obtained with a DA integration of the dynamics starting from the DA initial condition $\left[\boldsymbol{x}_{0}\right]=\boldsymbol{x}_{0}+\boldsymbol{\delta} \boldsymbol{x}_{0}$. Let us now consider the problem of computing an approximation of $\Phi(\boldsymbol{x}, \boldsymbol{q})$, in which we have highlighted explicitly the dependence of $\Phi$ on a vector of parameters $\boldsymbol{q}$. In the HOTM approach the only necessary adjustment in computing the approximation of $\Phi(\boldsymbol{x}, \boldsymbol{q})$ is to perform the DA integration in which also the parameters are initialized as DA variables, i.e. $[\boldsymbol{q}]=\boldsymbol{q}+\boldsymbol{\delta} \boldsymbol{q}$. The result is the HOTM $\mathcal{M}_{\boldsymbol{x}_{0}, \boldsymbol{q}}$ which can be now repeatedly evaluated to efficiently propagate, for many orbital revolutions, different initial conditions with different values of the parameters $q$.

In general $\mathcal{M}_{\boldsymbol{x}_{0}, \boldsymbol{q}}$ is nonlinear in $\boldsymbol{q}$ and thus it represents a good approximation of $\Phi(\boldsymbol{x}, \boldsymbol{q})$ in some neighborhood around $\boldsymbol{x}_{0}$ and $\boldsymbol{q}$. Furthermore, as each iterate of the map is also a 
function of $\boldsymbol{q}$, the evaluation of the map for values of the parameter far from the nominal one can quickly reduce its accuracy.

\subsection{HOTM accuracy estimation}

The main feature of the HOTM method is to increase the efficiency of orbital propagation. This is achieved by replacing computationally intensive numerical propagations with multiple evaluations of the HOTM map $\mathcal{M}_{\boldsymbol{x}_{0}}$. The main limitation of the approach is that the map can be used as long as it produces results with errors below an acceptable threshold $\epsilon_{\max }$.

If we define the maximum error of the HOTM method after $N$ iterates as

$$
\epsilon_{N}=\max _{i=1, \ldots, N}\left\|\widetilde{\boldsymbol{x}}_{i}-\boldsymbol{x}_{i}\right\|
$$

the map $\mathcal{M}_{x_{0}}$ can be used as long as $\epsilon_{N}<\epsilon_{\max }$. Overall, we can say that the accuracy is affected by the orbital regime, the perturbations included in (3) (which cause the deviation from Keplerian dynamics), the expansion order, and the total number of iterates $N$. In order for the HOTM method to be useful in practice it is required to obtain an estimate of the maximum number of revolutions $N_{\max }$ for which the map is sufficiently accurate, i.e. it is necessary to estimate the radius of convergence of $\mathcal{M}_{x_{0}}$.

In general it is difficult to a priori establish bounds on the radius of convergence. However, while computing the iterates $\widetilde{\boldsymbol{x}}_{i}$ there are relatively simple techniques to estimate the polynomial truncation error based on the size of the contribution from the highest order terms of $\mathcal{M}$ (Wittig et al. 2015). This method is based on the observation that in well converging polynomial expansions the higher order terms must converge towards zero. By evaluating only the highest order terms of the given expansion, and comparing their magnitude to that of the other lower-order terms, an estimate on the size of the truncation error can be made. A more sophisticated estimate involves the analysis of the size of the coefficients of each order, and fitting an exponential curve to the resulting values (Wittig et al. 2015). From this, a usually more accurate estimate of the truncation error and hence the convergence radius along each expansion variable could be obtained also a priori.

In this work, however, we forgo a detailed theoretical analysis of the convergence radius based on the analysis of the map $\mathcal{M}$. Instead, a heuristic approach to estimate the radius of convergence is adopted. This is based on running experiments for different orbital regimes, perturbations, and relevant spacecraft properties (mainly $\mathrm{A} / \mathrm{m}$ ) for fixed expansion orders. This allows us to define a look-up table with reasonable estimate of $N_{\max }$ for a prescribed accuracy. Our analysis is assessing the accuracy by comparing the results with fully numerical propagations.

In the case of non-autonomous perturbations the determination of the radius of convergence for the time variable, $r_{t}$, warrants an additional observation. Considering that typical non-autonomous perturbations, such as luni-solar perturbations, are sinusoidal perturbations of different periods, estimating $r_{t}$ analytically is possible. As an example, a Taylor expansion of the sine or cosine around 0 with expansion order 10 can cover approximately one period from $-\pi$ to $\pi$ with an absolute error of less than $1 \times 10^{-3}$. This means that for that accuracy, in the best case $r_{t}$ is on the order of a half-period of the perturbing force. In practice, however, $r_{t}$ is usually at least a factor of 2 smaller. This is due to the fact that the perturbing force is not only a simple trigonometric function, but a combination of trigonometric functions to describe the position of the body followed by further operations e.g. to compute inverse distances, which negatively affect the convergence properties. 
Table 1 Test case definitions

\begin{tabular}{|c|c|c|c|}
\hline $\begin{array}{l}\text { Test case } \\
\text { Reference mission }\end{array}$ & $\begin{array}{l}\text { A } \\
\text { QuickBird II }\end{array}$ & $\begin{array}{l}\text { B } \\
\text { ATV-5 }\end{array}$ & $\begin{array}{l}\mathrm{C} \\
\text { ESC-A } \\
\text { (ARIANE) }\end{array}$ \\
\hline Epoch (MJD2000) & 5337.16 & 5337.23 & 5195.16 \\
\hline$a(\mathrm{~km})$ & 6745.9 & 6792.3 & 24361.0 \\
\hline$e(-)$ & 0.001334 & 0.001877 & 0.7280 \\
\hline$i(\mathrm{rad})$ & 1.6936 & 0.9017 & 0.0517 \\
\hline$\Omega(\mathrm{rad})$ & 5.0697 & 3.1683 & 5.9375 \\
\hline$\omega(\mathrm{rad})$ & 2.6271 & 0.3751 & 3.0801 \\
\hline$A / m\left(\mathrm{~m}^{2} / \mathrm{kg}\right)$ & 0.0095 & 0.003 & 0.0115 \\
\hline$C_{D}(-)$ & 2.2 & 2.2 & 2.2 \\
\hline
\end{tabular}

\section{Test cases}

In the following section, we demonstrate the performance of the HOTM method by applying it to three different initial orbital conditions for which different perturbations become predominant. We select as the reference initial conditions those of the QuickBird II mission (labeled test case A), ATV-5 while in parking orbit (labeled test case B), and the ARIANE 5 upper stage ESC-A after the insertion of ASTRA 5B into a GTO (labeled test case C), as reported in Table 1. With this selection we can show the performances of the HOTM approach for a good range of orbital parameters (in particular for various semi-major axis, inclination and eccentricity). We use each test case to analyze in detail a specific aspect of the proposed method. In particular, test case A is used to study the performance of the approach when $J_{2}$ and drag perturbations are dominating. It also will be used to illustrate the effect of the computation order on the performance. Additionally, in test case B we study HOTM performances when considering continuously propelled low-thrust trajectories. Finally, the main focus in test case $\mathrm{C}$ is to assess the capability of the method to treat non-autonomous perturbations.

For each case, we first perform a full numerical integration of the system to establish what we consider the true trajectory. Then we employ the HOTM method to propagate the same initial condition. The results of the two methods are compared at the appropriate values of $L$ to compute the error after each revolution. For all test cases the HOTM is computed to order 5 in each spatial variable. Order 12 is used for expansion in the time variable in case of non-autonomous perturbations. For test case A also an analysis of the effect of the computation order on the accuracy and computational time is presented. All simulations are run on a MacBook Air with a $1.8 \mathrm{GHz}$ Intel i5 CPU and 4 GB RAM.

\subsection{Test case A: autonomous perturbations}

The reference orbit of test case A is a frozen, low-altitude, sun-synchronous orbit. The most significant perturbations in this case are Earth's oblateness $J_{2}$ and atmospheric drag.

Figure 5 shows the results of orbital propagation when only $J_{2}$ perturbation is accounted for. In the figures where the evolution of orbital parameters is analyzed we indicate with a grey line the results of the numerical integration, with a solid black line those of the same numerical integration evaluated at $L=0$, and finally a dashed line shows the results of the the HOTM (at $L=0$ by construction). 


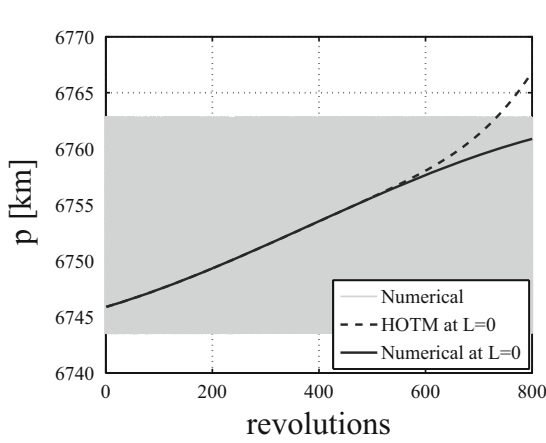

(a)

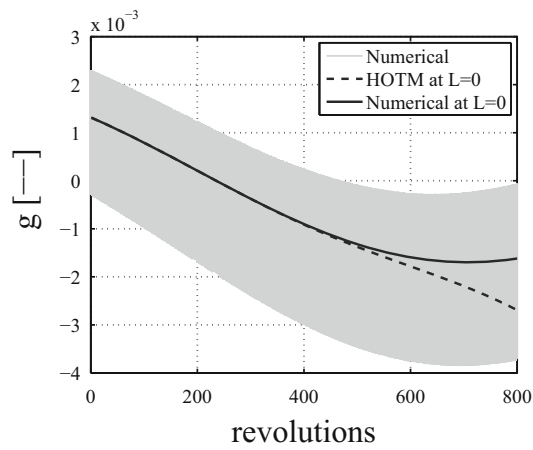

(c)

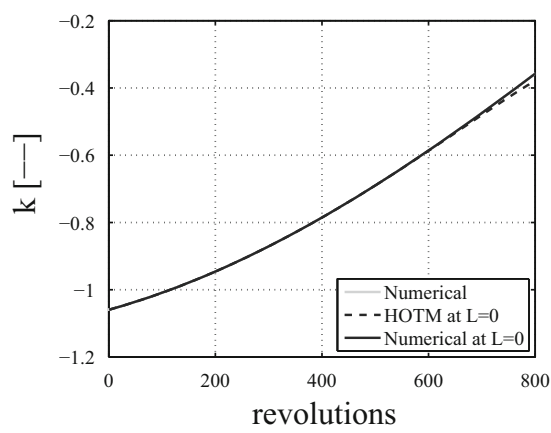

(e)

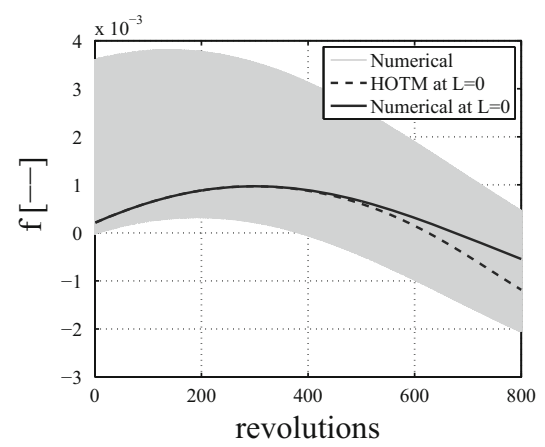

(b)

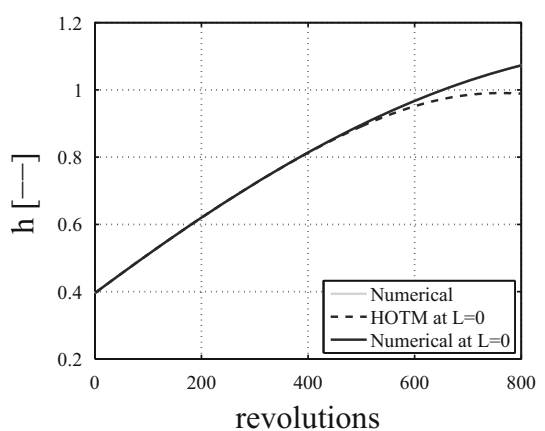

(d)

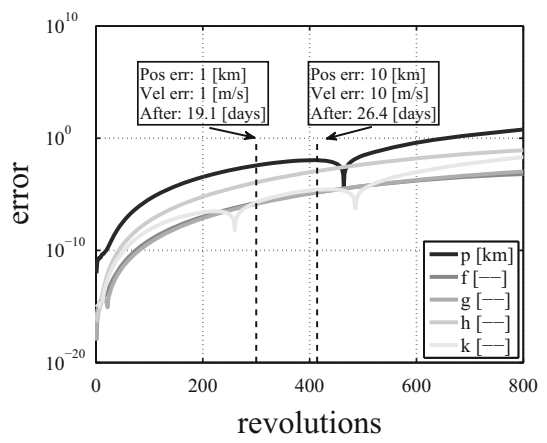

(f)

Fig. 5 Test case A: propagation under $J_{2}$ perturbation. a Semi-latus profile, b $f$ profile, $\mathbf{c} g$ profile, $\mathbf{d} h$ profile, e $k$ profile, $\mathbf{f}$ accuracy analysis

As expected, Fig. 5a highlights that when only $J_{2}$ perturbation is active there is no secular variation in the semi-latus rectum. Figure 5 shows the absolute error in each orbital variable as a function of the number of revolutions in logarithmic scale. As the physical meaning of the modified elements $p, f, g, h, k$ is not intuitively clear, the revolutions after which the the error in position and velocity exceeds for the first time a given threshold is also marked. The two pairs of thresholds chosen are $1 \mathrm{~km}$ and $1 \mathrm{~m} / \mathrm{s}$ respectively, as well as $10 \mathrm{~km}$ and $10 \mathrm{~m} / \mathrm{s}$. These thresholds can be considered representative as this is the range of positional 
Table 2 Overview of the validity of each HOTM and computational time comparison in the various cases

\begin{tabular}{|c|c|c|c|c|c|}
\hline \multirow[t]{2}{*}{ Test case } & \multirow[t]{2}{*}{ Perturbations } & \multicolumn{2}{|l|}{ Validity } & \multicolumn{2}{|c|}{ CPU sec (revs) } \\
\hline & & Days & Revs & HOTM & Num \\
\hline \multirow[t]{2}{*}{ A } & $J_{2}$ & $19.1-26.4$ & 299-414 & $1.77(500)$ & $9.56(500)$ \\
\hline & $J_{2}$, drag & $19.1-26.4$ & $299-414$ & $1.83(500)$ & $9.68(500)$ \\
\hline \multirow[t]{5}{*}{ B } & $J_{2}$, drag & $7.8-10.6$ & $122-165$ & $1.73(200)$ & $3.90(200)$ \\
\hline & Rad thrust & Always & Always & $0.26(4000)$ & $25.01(4000)$ \\
\hline & Tang thrust & $143-152.4$ & $1094-1121$ & $0.24(1100)$ & $8.77(1100)$ \\
\hline & $J_{2}$, drag, rad thrust & $7.8-10.6$ & $122-165$ & $1.78(200)$ & $4.02(200)$ \\
\hline & $J_{2}$, drag, tang thrust & $5.3-7.4$ & $81-112$ & $1.79(150)$ & $2.99(150)$ \\
\hline \multirow[t]{3}{*}{$\mathrm{C}$} & $J_{2}$, drag & $21-28.8$ & $49-67$ & $2.96(80)$ & $3.39(80)$ \\
\hline & $J_{2}$, drag, Sun & $18.8-25.8$ & $44-60$ & $16.95(80)$ & $5.27(80)$ \\
\hline & $J_{2}$, drag, Sun, Moon & $6.1-7.4$ & $15-18$ & $24.28(20)$ & $2.04(20)$ \\
\hline
\end{tabular}

errors associated to two line elements when evaluated at their corresponding reference epoch (Vallado et al. 2006).

These lines serve as an indication of the accuracy of the propagated orbit in real space. In particular, Fig. 5f shows that the approximation error is lower than $1 \mathrm{~km}$ and $1 \mathrm{~m} / \mathrm{s}$ for 299 revolutions (approximately 19.1 days), and lower than $10 \mathrm{~km}$ and $10 \mathrm{~m} / \mathrm{s}$ for 414 revolutions (approximately 26.4 days), as summarized in Table 2. Note that in both cases the first threshold to be violated is in the velocity while at that point the position error is still below the set threshold. The main contribution to the error stems from the $h$ and $k$ variables, which exhibit a total variation of $\approx 0.22$ and $\approx 0.18$ respectively over the first 299 revolutions. This gives a first idea of the convergence radius of these two variables in this orbital regime.

The computational time to compute the HOTM $\mathcal{M}$ is $1.77 \mathrm{~s}$ and a negligible $0.031 \mathrm{~s}$ is required for the propagation by evaluating $\mathcal{M}$ for 500 revolutions. The computational time to perform the same number of revolutions by numerical integration is $9.56 \mathrm{~s}$, as summarize in the Table 2. Note that the time for $\mathcal{M}$ evaluation is always negligible with respect to the time for building the map itself. As a result we can state that for HOTM approach the computational time does not depend on the propagation window, whereas for numerical propagation the computational time is proportional to the number of revolutions propagated. Thus, the more turns the map is valid for, the larger are the savings in computational time with respect to numerical propagation.

Figure 6 summarizes the most relevant changes to the results when also perturbation due to drag is accounted for. As shown in Fig. 6a the semi-latus is now correctly characterized by a secular decrease due to drag. It is interesting to note that the accuracy plot in Fig. $6 \mathrm{~b}$ is almost identical to that of Fig. 5f, and the validity of the HOTM method is exactly the same. This is due to the fact that the introduction of drag does not directly affect the $h$ and $k$ variables, as can be seen from Eq. 2. Thus, the error profiles for $h$ and $k$ are essentially the same as in the drag-free case, and the error threshold is violated after exactly the same number of orbital revolutions as in the previous case.

To illustrate the effect of the computation order on both the accuracy and the computational efficiency of the method, we performed the analysis on the case of $J_{2}$ perturbations only at various computation orders. Figure 7 shows the validity of the map for the $1 \mathrm{~km}, 1 \mathrm{~m} / \mathrm{s}$ and 


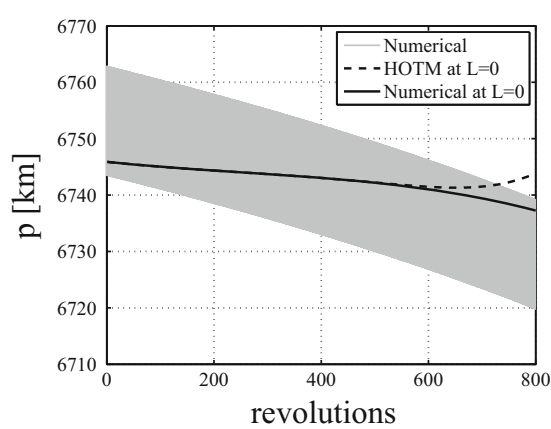

(a)

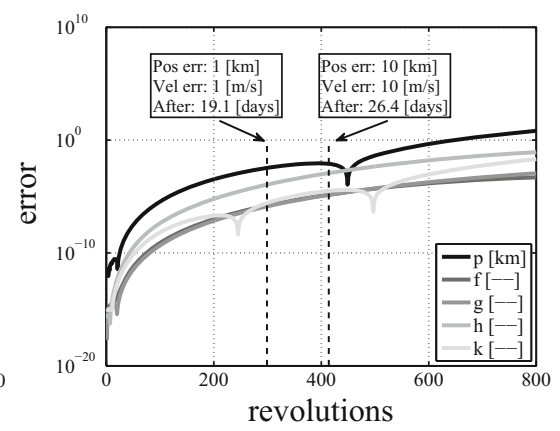

(b)

Fig. 6 Test case A: propagation under $J_{2}$ and drag perturbations. a Semi-latus profile, b accuracy analysis

Fig. 7 Test case A: dependence

of HOTM validity and computational time on computation order

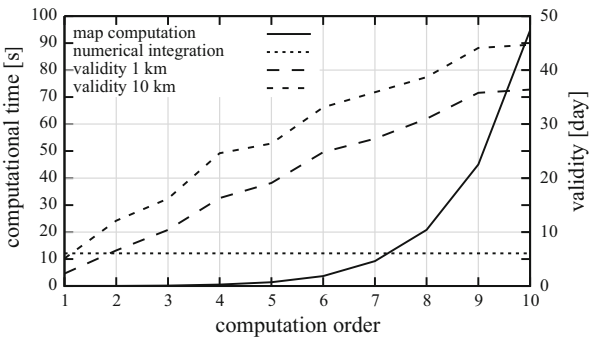

$10 \mathrm{~km}, 10 \mathrm{~m} / \mathrm{s}$ accuracy bounds along with the computational time required to compute the map as a function of the computation order.

It is evident that the computational time grows exponentially with the order, which is expected as the number of coefficients in each polynomial grows exponentially with the order. The validity of the map, meanwhile, in both cases increases about linearly with the order. For this case, we identify computation order 5 as the optimal order from the plot, where the trade-off between time to compute the map and the validity of the map is optimal. Depending on the specific requirements, also order 4 (faster computation, shorter validity) and order 6 (slower computation, longer validity) may be appropriate.

Going to higher orders becomes less efficient as the computational effort required to compute the HOTM grows too large. For reference, also the computational time for a purely numerical integration of the same orbit is shown. It is evident that around order 7 the break even point is reached, where the numerical integration becomes faster than the HOTM for the propagation of a single orbit. However, we remark that the HOTM, once computed, can easily be used to propagate similar initial conditions without additional computational cost.

\subsection{Test case B: low-thrust trajectories}

The initial conditions of the second test case represent a quasi circular orbit with inclination of $51.66 \mathrm{deg}$, i.e. the orbital inclination of the International Space Station which is the final target for the ATV-5 mission.

The first set of Fig. 8 shows the results of orbital propagation taking into account both $J_{2}$ and drag perturbations. Figure 8a clearly shows the secular decrease of the orbital energy and hence the semi-latus due to drag, as is expected for a near circular orbit such as this. By 
comparing Fig. 8f with Fig. 6b it becomes apparent that the validity of the HOTM is reduced from 299-414 to 122-165 revolutions (each pair of number corresponding to one of the two thresholds). The main reason of this validity reduction lies in the different inclination of the orbit which causes an increased variation of the $h$ and $k$ elements. In fact, considering their variation instead of the number of revolutions, it is clear that the change of $h$ of $\approx 0.1$ and that of $k$ of $\approx 0.3$ is of the same order as in test case A. Thus the convergence radius for both variables remains effectively unchanged, however their rate of change per revolution is now much larger and hence the number of revolutions is reduced. Also as in test case A, the velocity limit is the first one to be reached.

The main focus of this second test case is to show the performance of the HOTM method when continuously propelled trajectories are considered. Figure 9 shows the results when a radial thrust, providing a constant acceleration of $3 \times 10^{-4} \mathrm{~m} / \mathrm{s}^{2}$, is the only active perturbation. As expected from Eq. (2), the only orbital parameters affected by radial thrust are $f$ and $g$, while all remaining ones are constants of motion. After 4000 revolutions the accuracy of the HOTM is of order $1 \times 10^{-13}$ for both these variables, rendering the HOTM valid for virtually any number of revolutions. Truncation errors of the HOTM representation in this case are likely to be smaller than the numerical errors in the numerical integration and evaluation of the map.

In Fig. 10 the case with pure tangential thrust, with the same acceleration as for the radial case, is examined. The main effect of the thrust is to increase the orbital energy, as shown by Fig. 10a. It is remarkable how a single HOTM can accurately propagate a spiral trajectory for more than 140 days. The reason the errors increase after 1100 revolutions is not related to the HOTM method itself, but to the fact that at this point the dynamics for $L$ become singular. Figure 10b also shows that in this case the variable $p$ is responsible for reaching the HOTM validity limit. In fact note that the HOTM is considered valid until the error on $p$ reaches the thresholds of 1 and $10 \mathrm{~km}$ respectively.

Using the technique described in Sect. 4.4.2, the HOTM can be computed with the thrust magnitude as a variable parameter. This provides us with an analytical approximation of the dependence of the orbital parameters on the thrust magnitude. Such a HOTM can be profitably used to design orbit raising phases. In addition, the HOTM approach can be easily modified to include thrust laws that are anomaly dependent, thus further increasing the versatility of the method in mission design phases.

Finally, in Fig. $11 J_{2}$ and drag perturbations along with either radial and tangential thrust are considered. By comparing Fig. 11a with $8 \mathrm{f}$ it is clear that the introduction of radial thrust has no impact on map validity, which is driven by the change in $h$ and $k$ variables. On the other hand, the tangential thrust contributes to reduce the validity of the map from 122 to 165 revolutions to $81-112$, due to the increased errors in the $p$ variable.

\subsection{Test case $\mathrm{C}$ : non-autonomous perturbations}

The goal of the third test case is to assess the performances of the HOTM method in the presence of non-autonomous perturbations. For this reason a GTO, characterized by both high eccentricity and semimajor axis as well as low inclination, is selected. A full dynamical model is considered in this case, including the effect of $J_{2}$, drag, luni-solar gravitation perturbation, and solar radiation pressure. This represents the most critical dynamical framework for the HOTM method as time expansion is necessary to treat the non-autonomous perturbations.

In Fig. 12 the results achieved in terms of orbital parameters evolution and accuracy are depicted. As indicated by Fig. $12 \mathrm{f}$ the validity of the HOTM is only 15 orbital revolutions, which corresponds anyway to more than 6 days, due to the larger orbital periods compared 


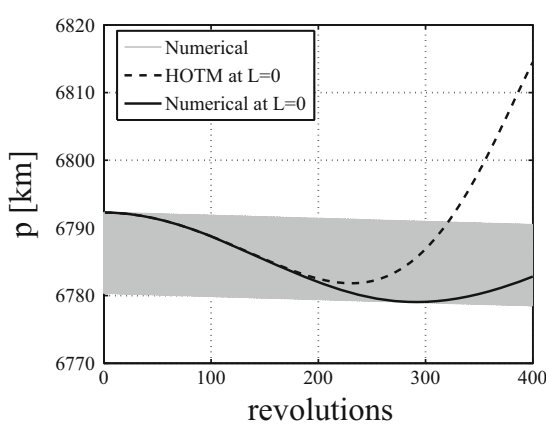

(a)

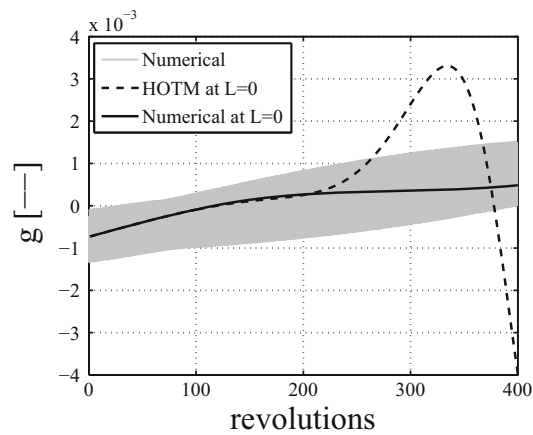

(c)

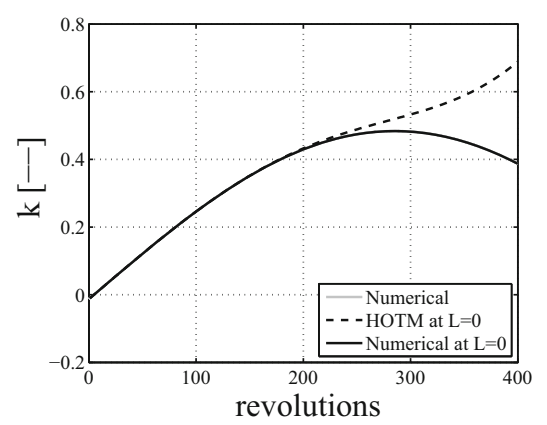

(e)

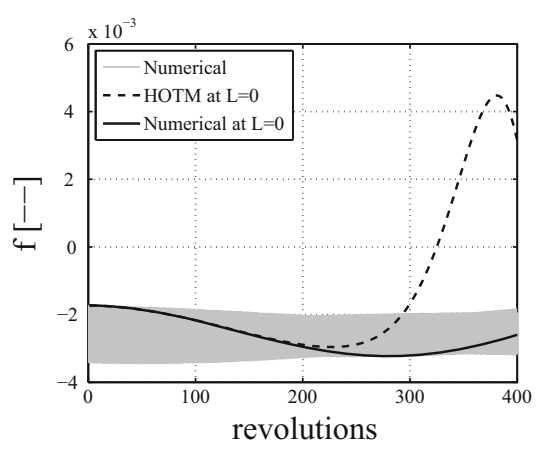

(b)

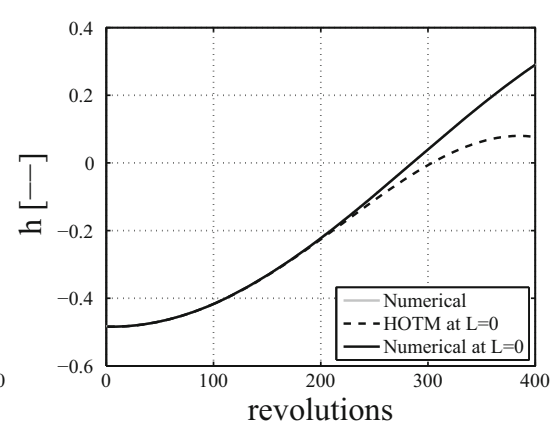

(d)

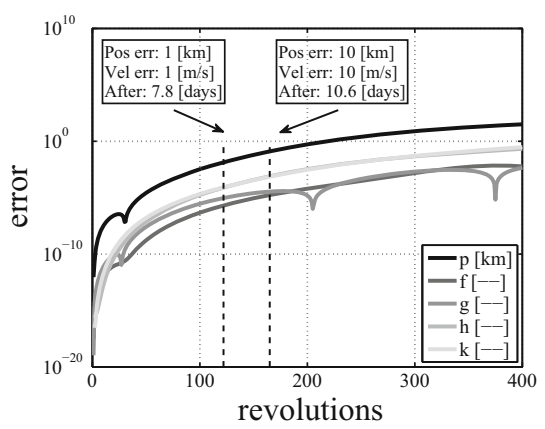

(f)

Fig. 8 Test Case B: propagation under $J_{2}$ and drag perturbations. a Semi-latus profile, $\mathbf{b} f$ profile, $\mathbf{c} g$ profile, d $h$ profile, e $k$ profile, $\mathbf{f}$ accuracy analysis

to test cases A and B. We remark that by choosing the time expansion point not at the initial time, as done here, but at the estimated radius of convergence as described in Sect. 4.5, the validity of the HOTM can essentially be doubled.

A validity of 6 days corresponds to a little under $1 / 4$ period of the Moon, which is the main factor in determining the radius of convergence in time for this map. Figure 13 serves to further support this analysis. When the perturbation of the Moon is neglected, and only solar perturbations are modeled, the validity of the HOTM jumps from 15 to 18 revolutions to $44-60$ as shown in Fig. 13a. If we finally also neglect the perturbations associated to 


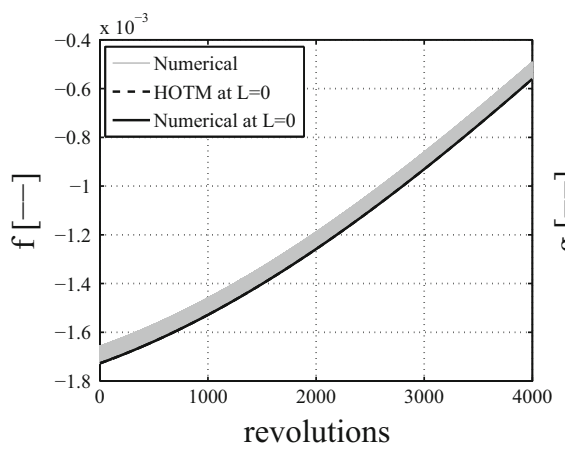

(a)

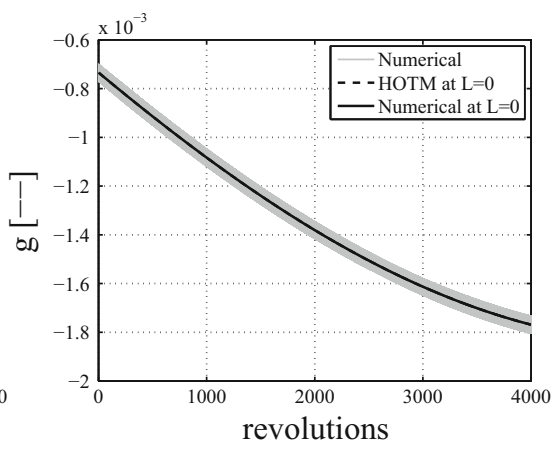

(b)

Fig. 9 Test case B: propagation with radial thrust only. a $f$ profile under radial thrust, $\mathbf{b} g$ profile under radial thrust

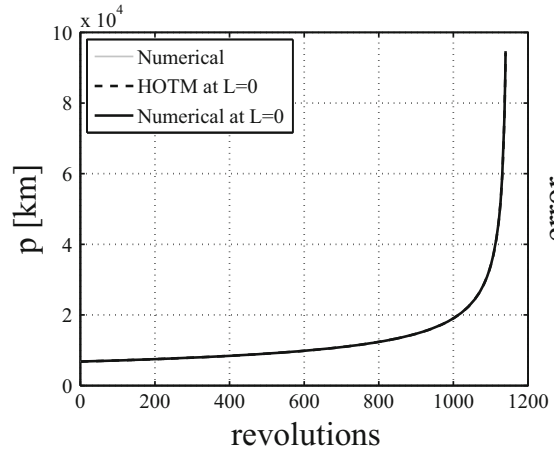

(a)

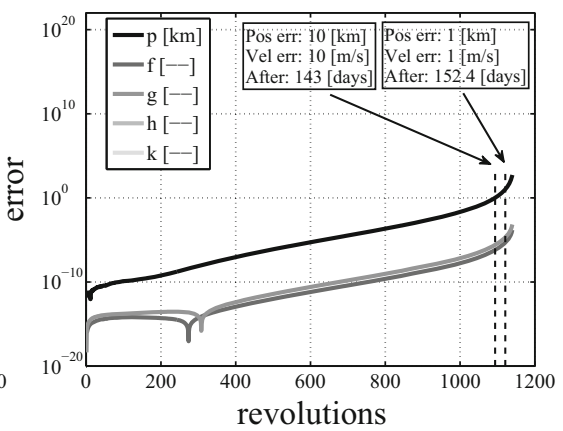

(b)

Fig. 10 Test case B: propagation with tangential thrust only. a $p$ profile under tangential thrust, b accuracy analysis under tangential thrust

the Sun, the validity of the HOTM extends to 49-67 revolutions. These findings confirm our previous analysis that in case of non-autonomous perturbations the time frequency of the perturbation is the key factor. The region of validity decreases drastically when nonautonomous perturbations with relatively high frequencies compared to the orbital period are included. Slow perturbations such as the Sun, on the other hand, have only a small impact on the validity of the HOTM.

As indicated in Table 2, when the full set of orbital perturbations is considered the computational time to propagate 20 revolutions with the HOTM method is $24.28 \mathrm{~s}$. This is mainly due to the need to expand the time variable up to order 12 . The computational time to perform the same number of revolutions by numerical integration is only $2.04 \mathrm{~s}$. As can be seen, in this case the map method for propagating a single initial condition is not able to recuperate the extra time spent in computing the high order time expansion compared to direct integration. However, the difference between both methods is only about a factor of 10 . Thus propagating a set of initial conditions (as required in many astrodynamics applications when uncertainties have to be taken into account) consisting of only 10 points already reaches the break-even point between both methods. Typically, the number of samples necessary to produce mean- 


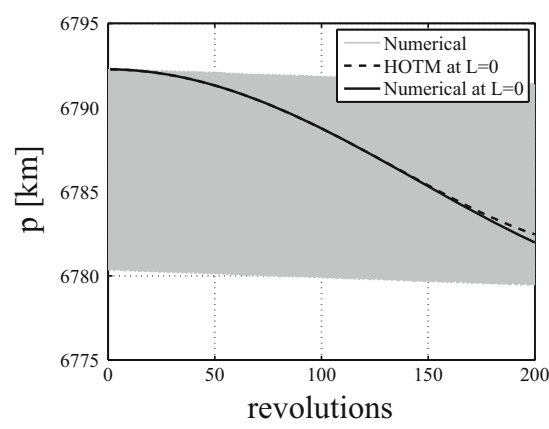

(a)

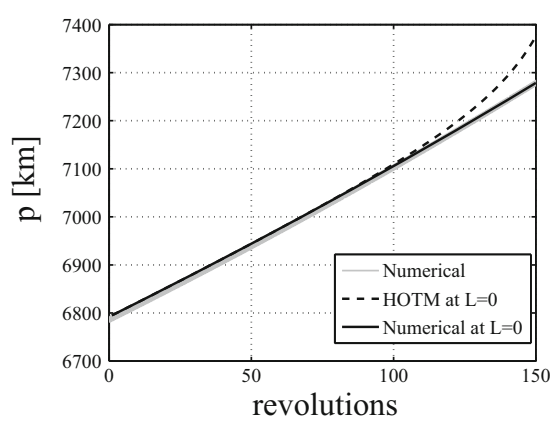

(c)

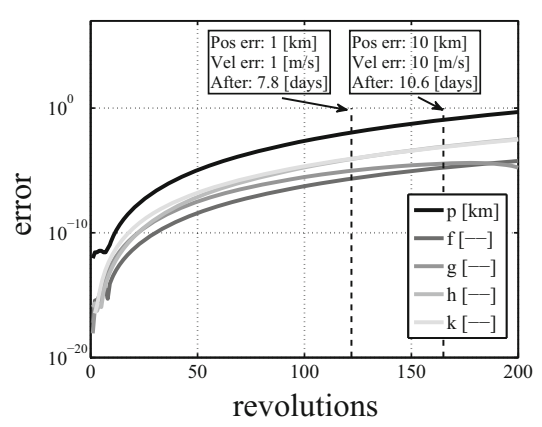

(b)

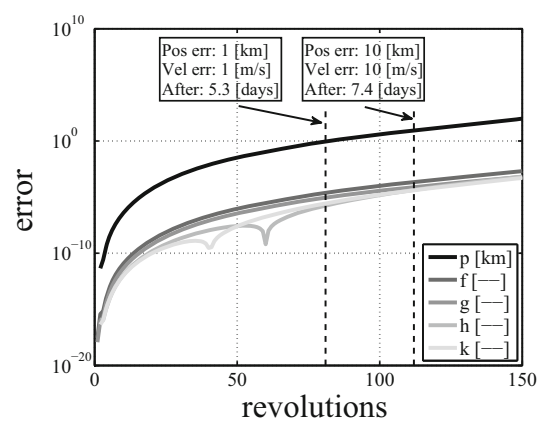

(d)

Fig. 11 Test case B: propagation under $J_{2}$, drag, and low-thrust perturbations. a Semi-latus profile under radial thrust,

b accuracy analysis under radial thrust, c semi-latus profile under tangential thrust, d accuracy analysis under tangential thrust

ingful statistical analysis are of course much larger, thus giving the advantage to the HOTM method in those cases.

\section{Conclusion}

The HOTM method introduced in this paper is a powerful tool for efficient orbit propagation in various orbital regimes subject to different perturbations. In many cases, particularly those with autonomous perturbations, the HOTM method is several times more efficient than the direct integration of the same dynamics even in the propagation of just a single orbit. Readers familiar with other works on DA methods will recognize that this is a remarkable result, as usually DA methods show their true potential only when used on a large number of initial conditions.

In the case of typical autonomous perturbations, the HOTM is valid for very long times, allowing the accurate propagation of orbits for hundreds if not thousands of revolutions. This includes the case of constant thrust both in radial and tangential direction. We demonstrated that the technique is still applicable to non-autonomous systems, as long as the frequency of the time-dependent perturbation is lower than the frequency of the quasi-periodic motion. 


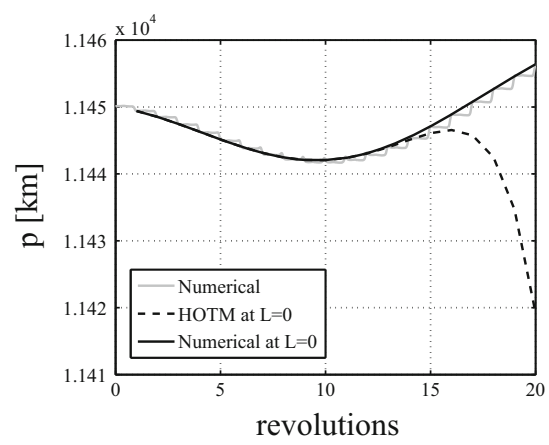

(a)

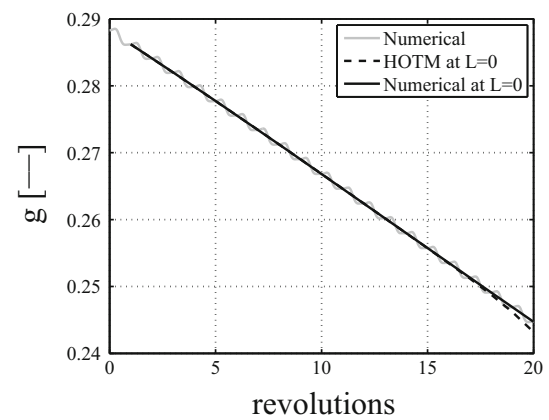

(c)

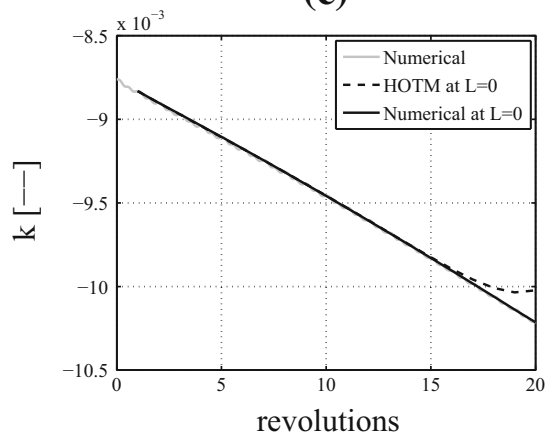

(e)

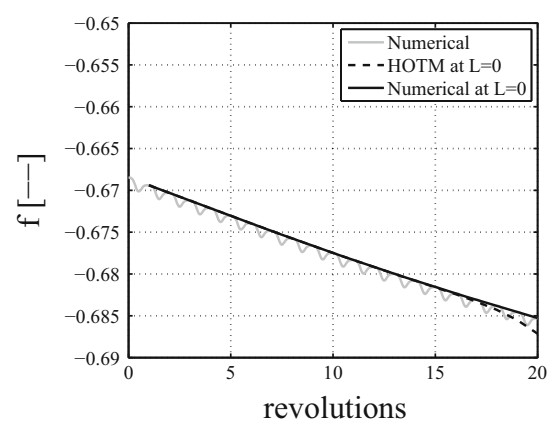

(b)

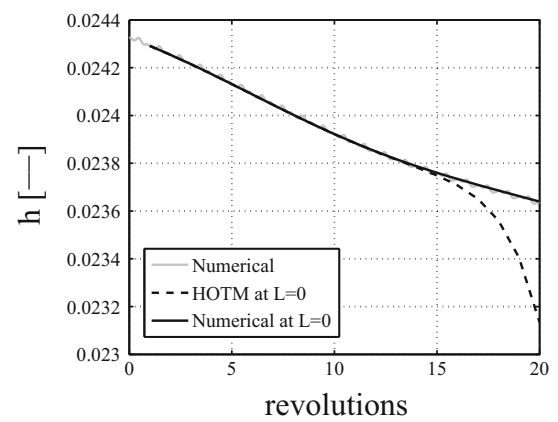

(d)

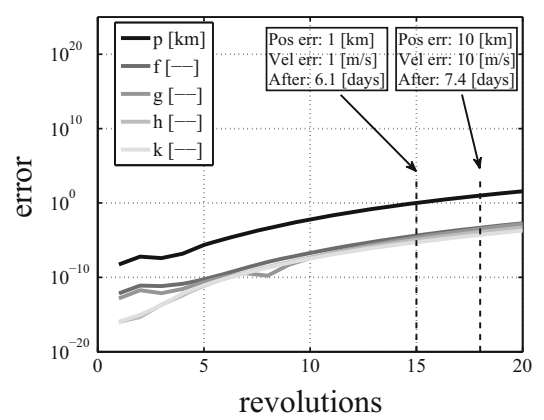

(f)

Fig. 12 Test case C: propagation in full dynamical model. a Semi-latus profile, b $f$ profile, $\mathbf{c} g$ profile, $\mathbf{d} h$ profile, e $k$ profile, f accuracy analysis

This includes typical non-autonomous perturbations such as luni-solar gravity as well as solar radiation pressure.

The examples shown in this work give an idea of the validity of the HOTM for different perturbations and orbital regimes. Table 2 gives an overview of the number of revolutions and the time for which the HOTM in each of the examples is valid.

Since the HOTM is only computed one single time by DA integration over one revolution, subsequent propagation of orbits reduces to the simple and fast evaluation of a polynomial map. Due to this, the time for even just the propagation of a single orbit is comparable to the 


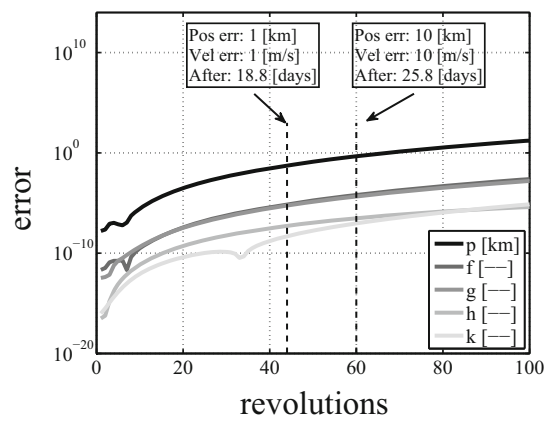

(a)

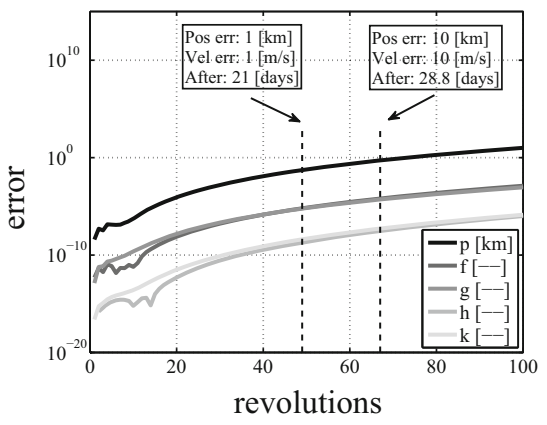

(b)

Fig. 13 Test case C: impact of the non-autonomous perturbations on HOTM validity. a Accuracy analysis under $J_{2}$, drag, and Sun perturbations, b accuracy analysis under $J_{2}$ and drag

time of direct numerical integration of the same single orbit. Considering the ability of the HOTM to propagate tens of thousands of initial conditions at once, e.g. in the propagation of a debris cloud, the transfer map method shows its true potential as the cost of the additional propagations is nearly zero. In such constellations, the method greatly outperforms pointwise integration as well as naive integration using DA for the full number of revolutions.

Acknowledgments R. Armellin is grateful to Dr. Martín Lara for having provided a comprehensive overview of orbital propagation methods. A. Wittig gratefully acknowledges the support received by the EU Marie Curie ITN AstroNet-II (PITN-GA 2011-289240) through an experienced researcher fellowship. The authors thank Dr. Martin Berz for introducing them to Differential Algebra techniques and his fundamental work in establishing many of the concepts applied in this work in the field of beam and accelerator physics. Furthermore, we are in debt to Pierluigi Di Lizia. This work can be considered as another fruit of the many discussions we had while working together. Once he finishes drilling on 67P/Churyumov-Gerasimenko, we look forward to working with him again.

\section{References}

Aksnes, K.: A second-order artificial satellite theory based on an intermediate orbit. Astron. J. 75, 1066-1076 (1970)

Berz, M.: The new method of TPSA algebra for the description of beam dynamics to high orders. In: Technical Report AT-6:ATN-86-16, Los Alamos National Laboratory (1986)

Berz, M.: The method of power series tracking for the mathematical description of beam dynamics. Nucl. Instrum. Methods A258, 431 (1987)

Berz, M.: Differential algebraic techniques. In: Tigner, M., Chao, A. (eds.) Handbook of Accelerator Physics and Engineering. World Scientific, New York (1999a)

Berz, M.: Modern Map Methods in Particle Beam Physics. Academic Press, London (1999b)

Berz, M., Makino, K.: COSY INFINITY version 9 reference manual. In: MSU Report MSUHEP-060803, Michigan State University, East Lansing, MI 48824, pp 1-84 (2006)

Bogoliuvov, N.N., Mitropolski, Y.A.: Asymptotic Methods in the Theory of Nonlinear Oscillations. Gordon and Breach, New York (1961)

Brouwer, D.: Solution of the problem of artificial satellite theory without drag. Astron. J. 64, 379-397 (1959)

Campbell, J.A., Jeffreys, W.H.: Equivalence of the perturbation theories of Hori and Deprit. Celest. Mech. 2, 467-473 (1970)

Deprit, A.: Canonical transformations depending on a small parameter. Celest. Mech. 1, 12-30 (1969)

Graf, O.F., Bettis, D.G.: Modified multirevolution integration methods for satellite orbit computation. Celest. Mech. Dyn. Astron. 11, 433-448 (1975)

Healy, L.M.: Orbit propagation with Lie transfer maps in the perturbed Kepler problem. Celest. Mech. 85, 175-207 (2003) 
Hoots, F.R., Roehrich, R.L.: Models for propagation of the NORAD element sets. In: Spacetrack Report no. 3, US Air Force Aerospace Defense Command, Colorado Springs, Col (1980)

Hori, G.: Theory of general perturbation with unspecified canonical variable. Publ. Astron. Soc. Jpn. 18, 287-296 (1966)

Kinoshita, H.: Third-order solution of an artificial satellite theory. In: Special Report No. 379, Smithsonian Astrophysical Observatory, Cambridge, Mass (1977)

Kozai, Y.: Second-order solution of artificial satellite theory without air drag. Astron. J. 67, 446-461 (1962)

Lara, M., San-Juan, J.F., López-Ochoa, L.M., Cefola, P.J.: On the third-body perturbations of high-altitude orbits. Celest. Mech. Dyn. Astron. 113, 435-452 (2012)

Long, A.C., Cappellari, J.O., Velez, C.E., Fuchs, A.J.: Goddard trajectory determination system (GTDS). Mathematical Theory Revision 1 CSC/TR-89/6001 (1989)

Lyddane, R.N.: Small eccentricities or inclinations in Brouwer theory of the artificial satellite. Astron. J. 68, 555-558 (1963)

McClain, W.D.: A recursively formulated first-order semianalytic artificial satellite theory based on the generalized method of averaging, volume 1: the generalized method of averaging applied to the artificial satellite problem. Computer Sciences Corporation CSC/TR-77/6010 (1977)

Métris, G., Exertier, P.: Semi-analytical theory of the mean orbital motion. Astron. Astrophys. 294, 278-286 (1995)

Montenbruck, O., Gill, E.: Satellite Orbits: Models, Methods, and Applications. Springer, Berlin (2000)

Morselli, A., Armellin, R., Di Lizia, P., Bernelli-Zazzera, F: A high order method for orbital conjunctions analysis: sensitivity to initial uncertainties. Adv. Space Res. 53, 490-508 (2014)

Picone, J.M., Hedin, A.E., Drob, D.P., Aikin, A.C.: NRLMSISE-00 empirical model of the atmosphere: statistical comparisons and scientific issues. J. Geophys. Res. Space Phys. 107, 1978-2012 (2002)

San-Juan, J.F.: ATESAT: automatization of theories and ephemeris in the artificial satellite problem. In: Tech. Rep. no. CT/TI/MS/MN/94-250, CNES, Toulouse, France (1994)

San-Juan, J.F.: ATESAT: review and improvements. Study of a family of analytical models of the artificial satellite generated by ATESAT and their numerical validation versus PSIMU and MSLIB. Tech. Rep. no. DGA/T/TI/MS/MN/97-258, CNES, Toulouse, France (1998)

Vallado, D.A., Crawford, P., Hujsak, R., Kelso, T.S.: Revisiting spacetrack report\# 3. AIAA J. 2006-6753, $1-88$ (2006)

Valli, M., Armellin, R., Di Lizia, P., Lavagna, M.: Nonlinear mapping of uncertainties in celestial mechanics. J. Guid. Control Dyn. 36, 48-63 (2013)

Walker, M.J.H., Owens, J., Ireland, B.: A set of modified equinoctial orbit elements. Celest. Mech. Dyn. Astron. 36, 409-419 (1985)

Walker, M.J.H.: Erratum—a set of modified equinoctial orbit elements. Celest. Mech. Dyn. Astron. 38, 391$392(1986)$

Wittig, A., Di Lizia P., Armellin, R., Makino, K., Bernelli-Zazzera, F., Berz, M.: Propagation of large uncertainty sets in orbital dynamics by automatic domain splitting. Celest. Mech. Dyn. Astron. (2015). doi:10. 1007/s10569-015-9618-3 